\title{
Rayleigh-Taylor instability in a sedimenting suspension
}

\author{
C. Völtz, W. Pesch, and I. Rehberg \\ Physikalisches Institut, Universität Bayreuth, D-95440 Bayreuth, Germany
}

(Received 11 July 2001; published 19 December 2001)

\begin{abstract}
The temporal evolution of an interface between glycerin and a glycerin-sand suspension of small packing fraction (obtained using the hindered settling phenomenon) driven by gravity is experimentally investigated. The growth rates for the different wave numbers characterizing the developing front are determined by means of a Fourier analysis. To model the observed behavior, we apply the idea of the Rayleigh-Taylor instability for a homogeneous fluid with vertically varying density and viscosity (one-fluid model). A good agreement between the experimental and theoretical results is obtained.
\end{abstract}

DOI: 10.1103/PhysRevE.65.011404

PACS number(s): 83.80.Hj, 47.54.+r, 47.20.Ma

\section{INTRODUCTION}

Sedimentation of granular grains in a fluid environment is a familiar phenomenon in nature and has important technical applications [1-4]. If the forces on the particles are dominated by the viscous interaction with the fluid, rather than by their inertia, a hydrodynamic description of the whole system has been proposed ([5-7], for more references see Ref. [8]). Such an approach demands confirmation in controlled experiments. A sensitive challenge for the hydrodynamic approach is certainly the dynamics of interfaces between regions in the fluid with different densities of the grains. The investigations might shed some light on the general understanding of interface dynamics in different branches of science.

In this paper, we consider the situation of sand particles immersed in highly viscous glycerin. One starts with a situation, where the sand covers the bottom of a vertically arranged Hele-Shaw-like cell. The cell is then turned upside down. The sand is thus layered above pure glycerin in a gravitationally unstable situation and starts sedimenting. The experiment is assumed to run in the case of prevailing viscous forces. Their relative importance is captured by the Bagnold number $B=\sqrt{\lambda} \rho_{\text {grain }} d^{2} \dot{\gamma} / \mu_{\text {fluid }}$, which expresses the ratio of collision forces between the grains to viscous forces in the fluid-grain mixture in a slightly different context, i.e., for gravity-free suspensions of solid particles in a uniform shear flow. Here $d$ is the diameter and $\rho_{\text {grain }}$ the density of the grains. The shear rate is denoted by $\dot{\gamma}$ and $\mu_{\text {fluid }}$ is the dynamic viscosity of the pure fluid. The parameter $\lambda$ introduced by Bagnold [9] is a measure for the concentration of the sand particles (i.e., the packing density). Commonly the description of a suspension as macroviscous is believed to be valid for Bagnold number $B<40$ [9]. In our experiment we have a Bagnold number of about $5 \times 10^{-5}$, thus the description of the glycerin-sand mixture as fluidlike seems to be conceptually well founded. In the experiments we were, in particular, interested in the instability of the initial flat glycerin-sand interface, which evolves into a fingerlike pattern. The Reynolds number $\mathrm{Re}$ $=\left(\rho_{\text {fluid }} v_{\text {finger }} d\right) / \mu_{\text {fluid }}$ in our experiments amounts to $4.2 \times 10^{-4}$ and the particle Reynolds number $\operatorname{Re}_{p}$ $=\left(\rho_{\text {fluid }} v_{\text {stokes }} d\right) / \mu_{\text {fluid }}$ amounts to $1.6 \times 10^{-5}$.

In our hydrodynamic approach, we model the fluid-sand system as a uniform fluid with macroscopically varying density. In the initial situation the heavier glycerin-sand mixture is stratified upon the lighter pure glycerin. The resulting fingering process is assumed to correspond closely to the wellknown Rayleigh-Taylor instability. To model the glycerinsand mixture as a Newtonian fluid with effective properties, depending on the concentration of the particles, is obviously a simplifying description. In such a model it is assumed that the particle concentration in the flowing mixture is almost constant. Furthermore, the particles have to be large enough to neglect their Brownian diffusion as in our experiments. Being aware of the possible limitations of a one-fluid model for the glycerin-sand mixture, we will examine whether such an approach can catch the essence of the experimental results.

The destabilization process of the interface is characterized by a collection of modes with different wave numbers $k$ and different growth rates $\sigma(k)$. In earlier experiments with water and polystyrene spheres [10] the authors concentrated on the dominant wave number (i.e. the fastest growing mode) seen in the early stages of the interface destabilization. One should also mention experiments with water and glass beads [11], which focus on the characterization of voidage shock fronts caused by a steep increase or decrease in the fluidization velocity for a fluidized bed.

One goal of the present investigation is to measure the whole spectrum $\sigma(k)$ for the wave numbers accessible in the experiment and to compare, in particular, to the theoretical results along the general lines of the Rayleigh-Taylor instability. A similar approach has already been performed for a suspension of a high initial packing density of $\phi=0.61$ of grains and with a sharp density interface [12]. The theoretical analysis based on a closed expression for $\sigma(k)$ for an idealized infinitely steep steplike interface gave a quite reasonable description of the experiments. However, the dynamic viscosity of the suspension was fitted. The resulting value, which was 104 times larger than the viscosity of the pure fluid, is difficult to assess in view of the conflicting relations between packing fraction and dynamic viscosity to be found in the literature [13-15].

In contrast, we will focus here on low packing fractions. The density profile is directly measured and used as input for the theoretical analysis. The dynamic viscosity can be calculated directly from an expression due to Einstein [16]. 
The paper is organized as follows. In the following section the experimental setup is described. In Sec. III the processing of the experimental data is detailed. The information is finally condensed into a $\sigma(k)$ relation, which is then compared to the theoretical results. Section IV describes the determination of the packing densities and of the shape of the density profile, which enter into theoretical analysis. The linear stability analysis is presented as well as further quantitative characterization of the growth rates. The final section contains the conclusions.

\section{EXPERIMENTAL SETUP}

In the experiments, a gravitationally unstable configuration of sand and glycerin is established by turning a HeleShaw-like cell upside down by means of a stepper motor, in a similar way as described in Ref. [12].

The interfacial instabilities in this paper develop from a fairly sharp, flat interface between regions of different packing densities. To prepare the initial situation the selfsharpening effect of hindered settling is exploited $[17,4,18]$. The clue is that the sedimentation velocity of a homogeneous suspension in a closed vessel decreases with increasing packing fraction. Thus a sharp interface, a shock, between the pure fluid and the sedimenting particles below the pure fluid is built. Then the vessel is rotated and the interface, now with glycerin and sand above pure glycerin, destabilizes.

To study the temporal evolution of a glycerin-sand interface driven by gravity a closed Hele-Shaw-like cell with a width of $2 \mathrm{~mm}$, a length of $98.0 \mathrm{~mm}$, and a height of $50 \mathrm{~mm}$ is used. The cell is filled with technical glycerin $(\sim 82 \%)$ as a carrier fluid and $2.0 \mathrm{~g}$ "sand."

The temperature during the whole measurements was stabilized to $(25.32 \pm 0.01)^{\circ} \mathrm{C}$ for several days. The standard deviation for any set of 201 measurements (see later) varies between $\pm 0.004{ }^{\circ} \mathrm{C}$ and $\pm 0.008{ }^{\circ} \mathrm{C}$.

The kinematic viscosity of the technical glycerin was measured with a viscometer to be $84.97 \mathrm{~mm}^{2} / \mathrm{s}$ at $T$ $=25.32{ }^{\circ} \mathrm{C}$ and was exactly evaluated for every set of measurements. The material density of the technical glycerin was determined with a density balance to be $1.25 \mathrm{~g} / \mathrm{cm}^{3}$ at these temperatures.

As "sand" we use spherical glass particles (Würth Ballotini MGL) with a material density of $2.45 \mathrm{~g} / \mathrm{cm}^{3}$. The particles are sieved in order to obtain a well-defined size distribution, which is then measured in a Coulter-counter (see Fig. 1). This measurement of the particle size distribution is based on the principle of monitoring the electrical current between two electrodes immersed in a conductive liquid on either side of a small aperture, through which the suspension of particles is forced to flow. As a particle passes through the aperture, it changes the impedance between the electrodes and produces an electrical pulse of short duration with an amplitude essentially proportional to the particle volume. An alternative measurement of the particle size distribution was based on light diffraction experiments. These measurements confirm the results in Fig. 1.

The cell is illuminated from behind by a field of LEDs that are run by dc current. The sand then appears bright in

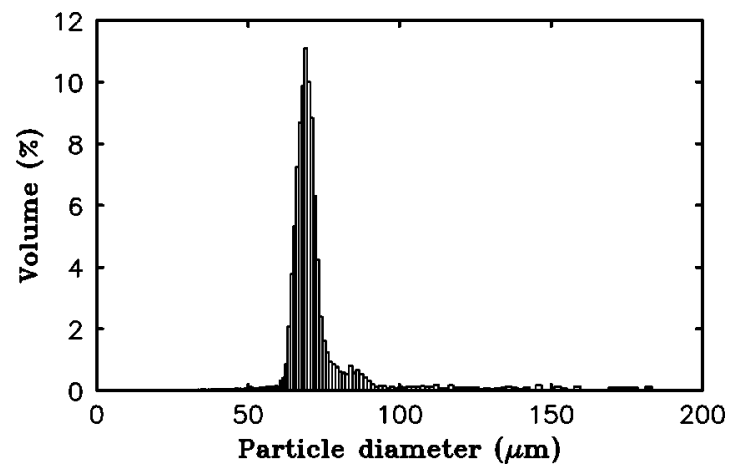

FIG. 1. Size distribution of the used particles, measured from conductivity measurements.

front of a dark background and can easily be detected by a charge coupled device camera connected to a frame grabber in the computer. The images have a dimension of $512 \times 350$ pixel. The optical resolution is given by $\Delta x=0.159 \mathrm{~mm} /$ pixel. From the contrast of the pictures, we obtain the packing fraction (see Sec. IV A), which yields the local density and the viscosity of the suspension.

To prepare the initial interface the cell is revolved twice by the use of a stepper motor. At the very beginning the cell is inserted into a frame when the sand is lying at the bottom of the cell. Then the cell is revolved within $233 \mathrm{~ms}$ so that the sand is layered above the glycerin. This is the starting point of continuously repeating cycles (201 times). The sand is sedimenting to the bottom of the cell and the selfsharpening effect forms a fairly sharp interface between the sedimenting suspension and the glycerin above. Before the sand has completed the sedimentation, the cell is revolved again after a varying waiting time $t_{w 1}$, so that the suspension comes to lie above the glycerin with an interface of which the instability is observed. The camera starts to take 55 snapshots, the first one $545 \mu$ s after the cell is turned. The time of the first snapshot defines the starting time $t=0$. The images are taken every second and show the middle part of the cell. After the images have been taken the cell is kept in its position for a constant waiting time $t_{w 2}=5 \mathrm{~min}$. Then the cell is rotated again and the cycle repeats. Figure 2 shows schematically the various stages of the experimental cycle.

We have been able to prepare well-defined distances of the interface from the vertical walls by keeping $t_{w 2}=5 \mathrm{~min}$ constant and varying $t_{w 1}$ in seven sets (each of 201 measurements) with $t_{w 1}=6,8,10,12,14,16$, and $20 \mathrm{~min}$. (For comparison, full sedimentation would require $t_{w 1} \approx 37 \mathrm{~min}$.) The interface instability becomes active twice in every cycle. Apparently an effective mixing of the sand and the glycerin takes place, where memory effects are wiped out. In fact, we observe the interfaces at constant $t_{w 1}$ always to form at the same height over the bottom of the cell. The averaged height $h_{a v}$ for every measurement is calculated by averaging the height of the interface in the lateral extension at $t=0 \mathrm{~s}$. Figure 3 shows the seven sets of different prepared heights of the interface above the bottom wall of the cell, each containing 201 single measurements. (For comparison, full sedimentation would result in an averaged height $h_{a v} \approx 43 \mathrm{~mm}$.) 


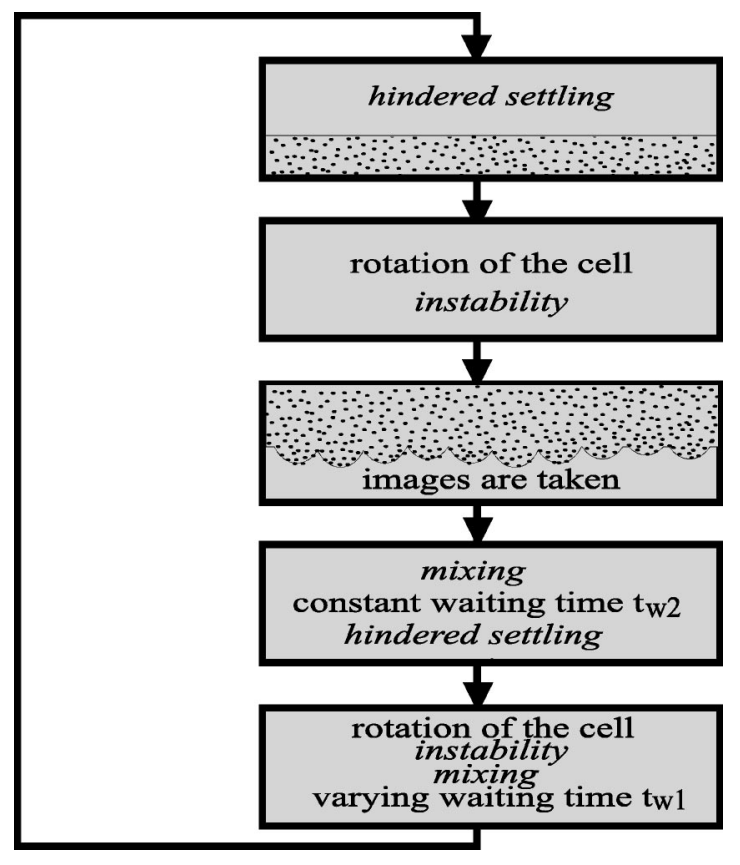

FIG. 2. Schematic procedure of the experimental cycle. By the different fonts the experimental preparation (normal font) and the system response (cursive font) are discriminated.

Since $h_{a v}$ for the first cycle has not yet reached the stationary level (as can be seen in Fig. 3) it is left out for the further evaluation of the data.

Obviously the averaged height $h_{a v}$ increases monotonically with increasing $t_{w 1}$. Figure 4 shows the dependence of the prepared height $h_{a v}$ as averaged over the remaining 200 measurements on the varying waiting time $t_{w 1}$.

\section{EXPERIMENTAL RESULTS}

As already mentioned the measurements of the growth rates have been performed for seven different series with

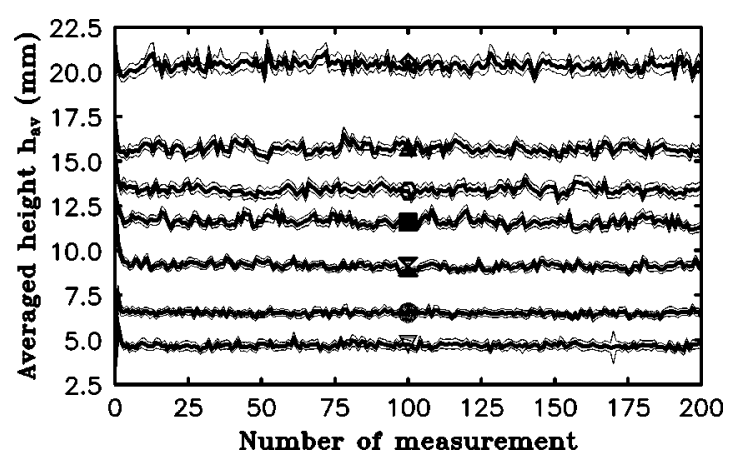

FIG. 3. Different interface heights above the bottom of the cell for seven series of measurements. The different symbols represent the different waiting times $t_{w 1}: 20 \mathrm{~min}$ (open rhombus), $16 \mathrm{~min}$ (filled triangle), $14 \mathrm{~min}$ (open hexagon), $12 \mathrm{~min}$ (filled square), 10 min (open hourglass), 8 min (filled circle), and 6 min (open triangle, upside down). The bold lines represent the measurements and the fine lines give the standard deviation of the averaging over all columns of an image. The symbols attached to the curves are the same as in Figs. 4, 9, 10, and 11.

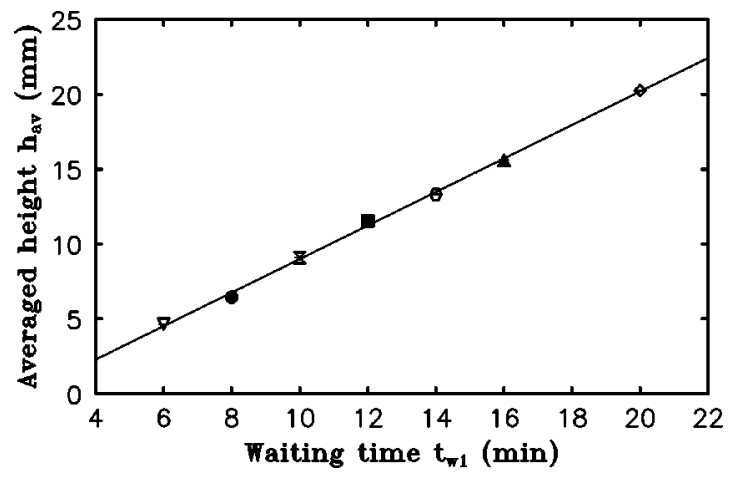

FIG. 4. Averaged height $h_{a v}$ as a function of $t_{w 1}$. The error bars that cannot be resolved in this plot, give the standard deviation. The symbols are the same as in Figs. 3, 9, 10, and 11.

different distances of the initial interface from the bottom of the cell (i.e., different waiting times $t_{w 1}$ ). Figure 5 shows a typical sequence of images during the development of the instability with the initial height $h_{a v}=9.1 \mathrm{~mm}, t_{w 1}$ $=10 \mathrm{~min}$.

To analyze the sequences, we apply a gradient algorithm involving suitable smoothing to pin point the glycerin-sand interface. The resulting interfaces are then passed through a low-pass filter keeping all wave lengths which are larger than 56 particle diameters. Figure 6 shows the temporal evolution of the images presented in Fig. 5.

In Fourier space one runs into the leakage problem when the offset between the heights at the limits of the windows is large. In order to avoid this problem the mismatch between the left and the right border of the interface is minimized by disregarding some (between 0 and 62) pixels at the edges.

Discrete Fourier transformation (DFT) of interfaces treated in this manner gives the Fourier spectrum of each interface. Figure 7 shows characteristic Fourier spectra of the interfaces at different times. Figure 8 shows the temporal evolution of the amplitude $A$ of a typical Fourier mode.

By an exponential fit

$$
A(k, t)=A_{i}(k) \exp (\sigma(k) t)
$$

we obtain the growth rate $\sigma(k)$ for every wave number $k$ in the Fourier spectra with $A_{i}$ the initial amplitude at $t=0$. The time window used in the fit procedure has been limited by two requirements: At first $A(k, t)(3.1)$ was kept smaller than $40 \%$ of the wavelength $2 \pi / k$, the criterion for the linear regime [19] and, in addition, $A(k, t)$ was kept smaller than half the height between the interface and the bottom of the cell.

As already explained before we have performed 200 cycles with a fixed set of parameters $\left(t_{w 1}, h_{a v}\right)$. These 200 measurements are analyzed to obtain a mean growth rate as a function of the wave number. The spectral resolution is $\Delta k$ $=2 \pi /(450 \Delta x)=0.88 \mathrm{~cm}^{-1}$. The ensuing dispersion relations are shown in Fig. 9. The lines are the theoretical results that will be explained in the next chapter. 

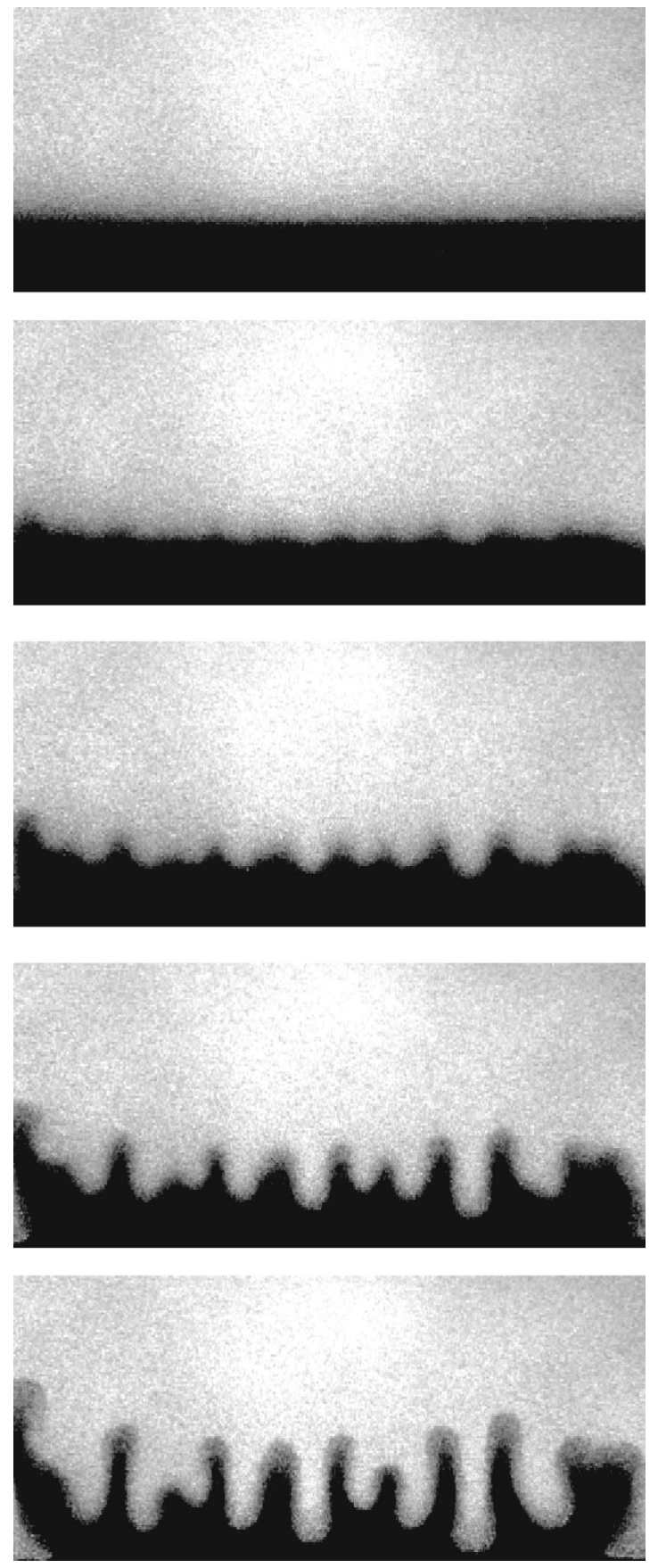

FIG. 5. Temporal evolution of the sand-glycerin interface at certain time steps: $0,30,35,40$, and $45 \mathrm{~s}$. The frames show the middle part of the cell and have a horizontal length of 512 pixels, respectively $81.39 \mathrm{~mm}$. The bright color represents the sandglycerin suspension, the dark color represents the glycerin. The contrast of the images is enhanced.

\section{THEORETICAL ANALYSIS AND COMPARISON WITH EXPERIMENTS}

\section{A. Determination of the packing fraction and density profile}

The vertical variations of the gray values of the images taken at $t=0$ are analyzed in terms of the packing density $\phi(z)$, which measures the volumetric concentration of the

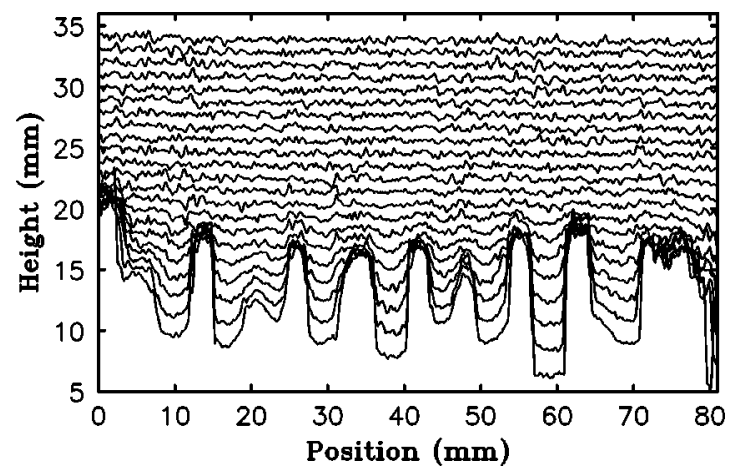

FIG. 6. Temporal evolution of the interface in Fig. 5 in the interval $0 \leqslant t \leqslant 42 \mathrm{~s}$ in steps of $2 \mathrm{~s}$. The patterns are shown with a constant vertical offset of $1 \mathrm{~mm}$.

sand particles in the mixture and from which the density $\rho(z)$ of the suspension can be calculated:

$$
\rho(z)=\phi(z) \rho_{\text {sand }}+[1-\phi(z)] \rho_{g l y c}
$$

with $\rho_{\text {sand }}=2.45 \mathrm{~g} \mathrm{~cm}^{-3}$ and $\rho_{\text {glyc }}=1.25 \mathrm{~g} \mathrm{~cm}^{-3}$. The packing density is directly measured in the experiments. It is always kept small in the experiments $(\leqslant 7 \%)$.

A nondimensionalized measure for the maximal density difference is the Atwood number $A=\left(\rho_{\text {sus }}-\rho_{\text {glycerin }}\right) /\left(\rho_{\text {sus }}\right.$ $\left.+\rho_{\text {glycerin }}\right)=0.03$ for $\rho_{\text {sus }}=\rho(z)$ with $\phi(z)=0.065$.

The calibration between the gray values and the packing density is achieved by preparing suspensions of homogeneous packing fractions by repeatedly rotating the cell and averaging the measured gray values. From this calibration the actual packing fractions and density profiles are known. The mean density profiles are smoothed with a cubic spline algorithm and enter directly into the theoretical description (see Sec. IV B). The density profiles for the different measurements are shown in Fig. 10.

\section{B. Linear stability analysis}

The pattern forming process displayed in Fig. 5 starts from a situation (top panel), where the concentration of the suspended sand particles and consequently the density of the glycerin-sand mixture decreases monotonically towards the

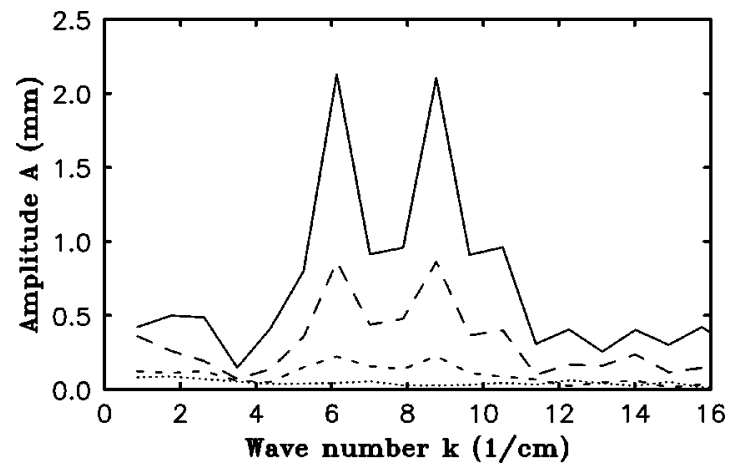

FIG. 7. Fourier spectrum of the interface evolution at times $t=0$ $\mathrm{s}$ (dotted line), $30 \mathrm{~s}$ (short-dashed), $35 \mathrm{~s}$ (long-dashed), and $40 \mathrm{~s}$ (solid line). The data belong to the interface presented in Fig. 6. 




FIG. 8. Amplitude $A$ of a DFT analysis for a typical wave number (here $k \sim 6.1 \mathrm{~cm}^{-1}$ ) in dependence on time $t$. An exponential fit is obtained. The data belong to the interfaces presented in Fig. 6 .

bottom of the Hele-Shaw-like cell. Due to gravity this configuration is mechanically unstable. Theoretically the fairly sharp planar interface (see Fig. 10) might continuously move downward until the sand covers the bottom as a dense package. However, the interface becomes obviously unstable with increasing time and develops fingerlike protrusions (Fig. 5), which we will analyze in the following. In a hydrodynamic approach the system is modeled as a homogeneous onecomponent fluid with variable density. In the initial state the fluid is horizontally stratified, with a heavier layer (bright in Fig. 5) on top of a lighter one (black). Thus, we make contact to the well-known Rayleigh-Taylor instability according to which the destabilization of the interface between the layers is inevitable.

In our analysis, we will closely follow the standard approach to be found in Chandrasekhar's authoritative work [20]. A Cartesian coordinate system is used in the cell. It extends from $z=0$ to $z=d$ in the vertical direction (antiparallel to gravity) and is idealized as infinitely extended in the span-wise $x$ direction. The narrow gap covers the interval $-a / 2 \leqslant y \leqslant a / 2$. The starting configuration is characterized by the density profile $\rho(z)$ and a pressure $p(z)$. Its linear stabil-

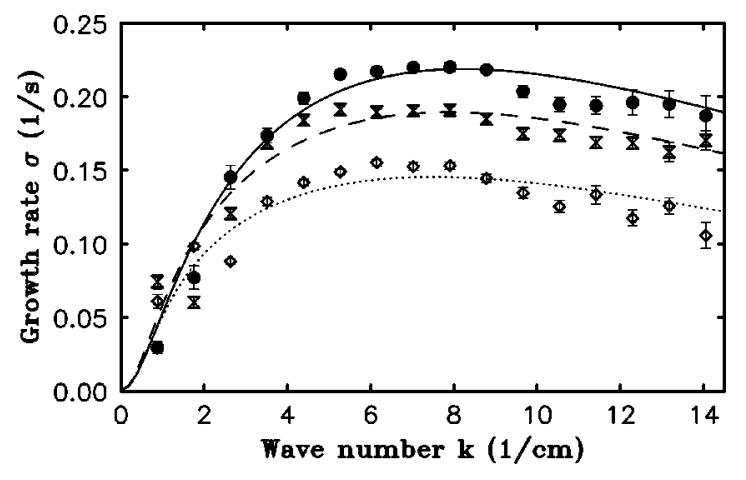

FIG. 9. Comparison of experimental data (single points) with the Rayleigh-Taylor model [lines from Eq. (4.2)] for a onecomponent fluid with vertically varying density and viscosity. For details see Sec. IV B and IV C. The solid line corresponds to the filled circles, the dashed line to the open hourglasses, and the dotted line to the open rhombi. The error bars give the standard deviation of the mean value. The symbols are the same as in Figs. 3, 4, 10, and 11 .

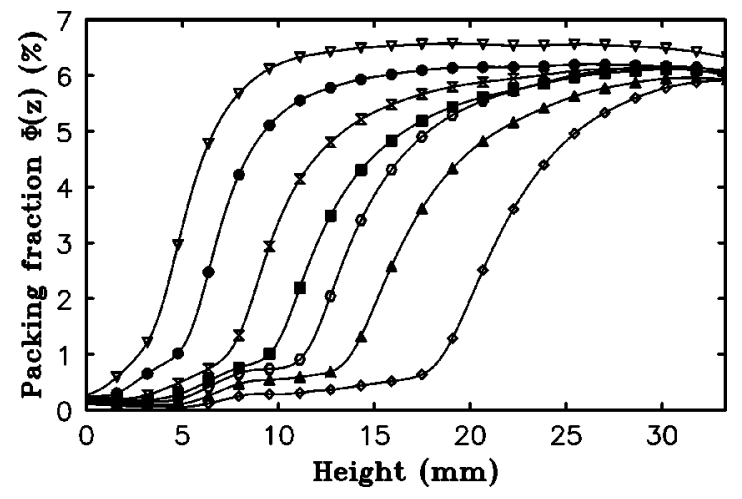

FIG. 10. Density profiles of different measurements, i.e., different $t_{w 1}$. The profiles show different steepness and different distances to the vertical walls. They were smoothed with a cubic spline algorithm. The symbols are the same as in Figs. 3, 4, 9, and 11.

ity against density fluctuations $\delta \rho$, pressure fluctuations $\delta p$ and the ensuing velocity field $\mathbf{u}$ is calculated via the suitably linearized Navier-Stokes equations (see Ref. [20])

$$
\begin{gathered}
\rho \partial_{t} \mathbf{u}=-\nabla \delta p+\nabla \cdot \Sigma-G \delta \rho \mathbf{e}_{z}, \\
\nabla \mathbf{u}=0, \quad \partial_{t} \delta \rho+(\mathbf{u} \cdot \nabla) \rho(z)=0 .
\end{gathered}
$$

The components of the stress tensor $\Sigma$ are given as

$$
\Sigma_{i, j}=\mu(z)\left(\partial_{i} u_{j}+\partial_{j} u_{i}\right) ; \quad i, j=(x, y, z) .
$$

$G$ denotes the gravity constant and $\mu(z)$ the dynamical viscosity. The density $\rho(z)$ of the suspension is determined by $\phi(z)$ according to Eq. (4.1). The viscosity $\mu$ is also given in terms of $\phi(z)$ by the following relation valid for small $\phi$ [16]:

$$
\mu(z)=\mu_{g l y c}[1+2.5 \phi(z)] .
$$

The viscosity $\mu_{g l y c}$ of our carrier fluid, technical glycerin, has been measured directly as function of temperature.

In the following we adopt an approximation scheme, originally proposed by Brinkman [21], that has proven to be adequate for a narrow slab geometry in a similar context (see for instance the discussion in Ref. [22]). The main idea is to keep in Eq. (4.2) only the spatial variations in the $x, z$ plane by appropriately averaging out the variations in the $y$ direction. This procedure is expected to be reliable as long as the fingers remain two dimensional in the narrow gap case. The $y$ component of the velocity perpendicular to the slab is certainly very small and is consequently neglected. The remaining $x, z$ components of $\mathbf{u}$, which have to vanish at $y= \pm a / 2$ are then averaged over the $y$ direction.

The incompressibility condition $\boldsymbol{\nabla} \mathbf{u}=0$ is ensured by the introduction of a velocity potential $f$ :

$$
\mathbf{u}=\vec{\delta} f(x, z, t), \quad \vec{\delta}=\left(\partial_{x z}^{2}, 0,-\partial_{x x}^{2}\right) .
$$

After taking the scalar product of Eq. (4.2) with $\vec{\delta}$ one arrives at an equation for $f$; the pressure drops out.

To perform the $y$ average, we use the following ansatz: 


$$
\begin{gathered}
f(x, z, y, t)=\exp (i k x+\sigma t) \tilde{f}(z) p(y), \\
\delta \rho(x, y, z, t)=\exp (i k x+\sigma t) \tilde{\rho}(z) p(y) .
\end{gathered}
$$

The smooth profile $p(y)$ that vanishes at $y= \pm a / 2$ captures the $y$ dependence of all fields. It is conveniently characterized by the "mean curvature" $c$ defined as

$$
c=-\int_{-a / 2}^{a / 2} d y \frac{d^{2}}{d y^{2}} p(y) / \int_{-a / 2}^{a / 2} d y p(y) .
$$

For $p(y)=\cos (\pi y / a)$, we obtain $c=(\pi / a)^{2}$ and for the Hagen-Poisseuille profile $p(y)=-\left[y^{2}-(a / 2)^{2}\right]$ the value $c$ $=12 / a^{2}$.

Inserting the ansatz (4.6) into Eq. (4.2) a linear eigenvalue problem is obtained that yields the growth rate $\sigma=\sigma(k)$ as a function of $k$ :

$$
\begin{aligned}
\sigma\left[D(\rho D \tilde{f})-\rho_{0} k^{2} \tilde{f}\right]= & D\left[(D \mu)\left(D^{2}+k^{2}\right) \tilde{f}\right. \\
& +\mu\left[\left(k^{2}-D^{2}-c\right) \tilde{f}\right]+2 k^{2}(D \tilde{f})(D \mu) \\
& -D\left[\mu\left(D^{2}-k^{2}-c\right)\right] \tilde{f}-g \delta \tilde{\rho}, \\
& \sigma \widetilde{\rho}=k^{2} D \rho(z),
\end{aligned}
$$

with $D=d / d z$. Note that Eq. (4.8) for $c=0$ is identical to the corresponding system in Ref. [20], where a large extension in the $y$ direction had been assumed.

The eigenvalue problem Eq. (4.8) has to be solved with the no-slip boundary conditions $\widetilde{f}=D \widetilde{f}$ at $z=0, d$ and $\mathrm{c}$ fixed. For general $\rho(z), \mu(z)$ the growth rates have then to be determined numerically; they are shown for a suitably determined $c$ (see below) as function of $k$ in Fig. 9 .

\section{Quantitative characterization of the growth rates}

We have calculated the $\sigma(k, c)$ from Eq. (4.8) for a series of curvatures $c$ and to evaluate $\chi^{2}(c)=\left[\sigma_{\text {exp }}(k)-\sigma(k, c)\right]^{2}$ for the corresponding experimental points shown in Fig. 9 for $k \leqslant 14.1 \mathrm{~cm}^{-1}$. By fitting $\chi^{2}(c)$ to a parabola about its minimum $c=c_{\text {opt }}$,

$$
\chi^{2}(c)=a\left(c-c_{o p t}\right)^{2}+\chi_{o p t}^{2}
$$

we obtain the optimal mean curvature $c_{\text {opt }}$ with minimal $\chi^{2}$. These curvatures $c_{\text {opt }}$ were used for calculating the theoretical growth rates $\sigma\left(k, c=c_{o p t}\right)$. The three selected examples shown in Fig. 9 correspond to $c_{\text {opt }}=11.48 / a^{2}, \quad c_{\text {opt }}$ $=11.52 / a^{2}$, and $c_{o p t}=13.33 / a^{2}$ from above. The values of $c_{\text {opt }}$ for all seven measurements can be found in Fig. 11(e). All values are near to $c=12 / a^{2}$, corresponding to the Poiseuille profile.

By a fit of the experimental $\sigma_{\exp }(k)$ to

$$
\sigma_{\text {exp }}(k)=a\left(x-k_{\max }\right)^{2}+\sigma_{\max }
$$

for $3.51 \leqslant k \leqslant 10.54$, we obtain the maximal growth rate $\sigma_{\max }$ and the wave number of maximal growth rate $k_{\max }$.



FIG. 11. $\Delta \phi, \kappa, \sigma_{\max }, k_{\max }$, and $c$ as a function of the height $h_{a v}$. The symbols are the same as in Figs. 3, 4, 9, and 10.

Both parameters show a systematic dependence on the distance from the wall, to be seen in Fig. 11(c) and 11(d). For smaller distances over the bottom of the cell $k_{\text {max }}$ increases since the distance decreases and hence the wavelength.

$\sigma_{\max }$ increases for small distances as well. A possible explanation could be that the profiles are steeper near the bottom of the cell, as seen in Fig. 10. The steepness $\kappa$ can be quantified by a tanh-fit

$$
\phi(z)=\Delta \phi \tanh [\kappa(z-b)]+\phi_{0}
$$


which yields also $\Delta \phi$, the difference of packing density between top and bottom of the cell. These quantities show the expected decrease with increasing height $h_{a v}$ over the bottom of the cell [Fig. 11(a) and 11(b)].

A close correlation between $\kappa, \Delta \phi, \sigma_{\max }, k_{\max }$, and the distance to the bottom wall or $t_{w 1}$, respectively, is obvious.

There seems to be a deviation between the experimental and the theoretical growth rates for small $k$. This might be due to the fact that the experiment is finite in the $x$ direction in contrast to the theoretical idealization.

One sees further, that the theoretical growth rate $\sigma(k)$ decreases more slowly as function of $k$ compared to the experimental results. A possible explanation could be, that at larger $k$ the diffusion of the sand particles gets more relevant, which we have neglected in our approach. Diffusion would effectively decrease the initial steepness of the density profile in time and consequently lead to smaller effective growth rates.

\section{CONCLUSION}

In a closed Hele-Shaw-like cell the temporal evolution of a glycerin-sand interface was investigated. For the unstable stratification, a suspension of sand and glycerin above glycerin, the instability is driven by gravity. The images of the temporal evolution were analyzed by DFT. The Fourier spectra show that the initial disturbances of the interface grow exponentially at the beginning of the pattern forming pro- cess. This enables us to determine the growth rates by an exponential fit for every wave number $k$ in our spectra. The data show that the growth rate has a nonmonotonic $k$ dependence: It increases steeply towards a maximum at $k \sim 7$ $\mathrm{cm}^{-1}$. Then it decreases moderately again.

To describe the general behavior we have chosen a onefluid model with vertically varying density. Carrying out a linear stability analysis for the interface we calculate the growth rates from the dispersion relation.

The analysis of the dependence of $\sigma_{\max }$ and $k_{\max }$ on the height over the bottom of the cell shows a systematic behavior, as well as the dependence of $\Delta \phi$ and $\kappa$.

The continuum approach gives a good agreement with the experimental results: the one-fluid model describes satisfactorily the experimental results. Thus, our present results show convincingly that a simple hydrodynamic approach to diluted suspensions is reasonable. More refined theoretical models like two-fluid models [5] and [6] seem to yield no additional insight for the low concentration suspension used in our experiment.

\section{ACKNOWLEDGMENTS}

It is a pleasure to thank Friedrich Busse, Lutz Heymann, Adrian Lange, and Matthias Schröter for stimulating discussions and technical support. The experiment was supported by Deutsche Forschungsgemeinschaft through Re588/12.
[1] M. Ungarish, Hydrodynamics of Suspensions (Springer, New York, 1993).

[2] J.M. Burgers, Proc. K. Ned. Akad. Wet. 44, 1045 (1941); 45, 9 (1942).

[3] C.W.J. Beenakker, W. van Saarloos, and P. Mazur, Physica A 127, 451 (1984); C.W.J. Beenakker and P. Mazur, Phys. Fluids 28, 767 (1985); Physica A 131, 311 (1985); Phys. Fluids 28 (11), 3203 (1985).

[4] R.H. Davis and A. Acrivos, Annu. Rev. Fluid Mech. 17, 91 (1985).

[5] B. Noetinger, Physica A 157, 1139 (1989).

[6] R.-H. Jean and L.-S. Fan, in The Handbook of Fluid Mechanics, edited by R.W. Johnson (Springer, Boca Raton, FL, 1998), Chap. 19.

[7] A. Lange, M. Schröter, M.A. Scherer, A. Engel, and I. Rehberg, Eur. Phys. J. B 4, 475 (1998).

[8] Sedimentation of Small Particles in a Viscous Fluid, edited by E.M. Tory (Computational Mechanics Publications, Southampton, England, 1996).

[9] R.A. Bagnold, Proc. R. Soc. London, Ser. A 225, 49 (1954).

[10] A.T. Skjeltorp, in Time-Dependent Effects in Disordered Materials, edited by R. Pynn and T. Riste (Plenum Press Corporation, New York, 1987), p. 71.
[11] A.K. Didwania and G.M. Homsy, Ind. Eng. Chem. Fundam. 20, 318 (1981). We are referring especially to the experimental runs, where an initially unsaturated bed of beads was suddenly exposed to a high velocity fluid.

[12] C. Völtz, M. Schröter, G. Iori, A. Betat, A. Lange, A. Engel, and I. Rehberg, Phys. Rep. 337, 117 (2000).

[13] I.M. Krieger and T. J. Dougherty, Trans. Soc. Rheol. 3, 137 (1959).

[14] J.S. Chong, E.B. Christiansen, and A.D. Baer, J. Appl. Polym. Sci. 15, 2007 (1971).

[15] N.A. Frankel and A. Acrivos, Chem. Eng. Sci. 22, 169 (1967); A. Acrivos in Particulate Two-Phase Flow, edited by M.C. Rocco (Butterworth-Heinemann, Boston, 1993), p. 169.

[16] A. Einstein, Ann. Phys. (Leipzig) 19, 289 (1906); 34, 591 (1911).

[17] G.J. Kynch, Trans. Faraday Soc. 48, 166 (1952).

[18] R.H. Davis and M.A. Hassen, J. Fluid Mech. 196, 107 (1988).

[19] D.J. Lewis, Proc. R. Soc. London, Ser. A 202, 81 (1950).

[20] S. Chandrasekhar, Hydrodynamic and Hydromagnetic Instability (Dover, New York, 1981).

[21] H.C. Brinkman, Appl. Sci. Res., Sect. A 1, 27 (1947).

[22] J. Huang and B.F. Edwards, Phys. Rev. E 54, 2620 (1996). 